\title{
TRIÁNGULOS EN NUESTRO ENTORNO Y SU CLASIFICACIÓN
}

\author{
AUTORES: Erika María Muñoz Delgado ${ }^{1}$
}

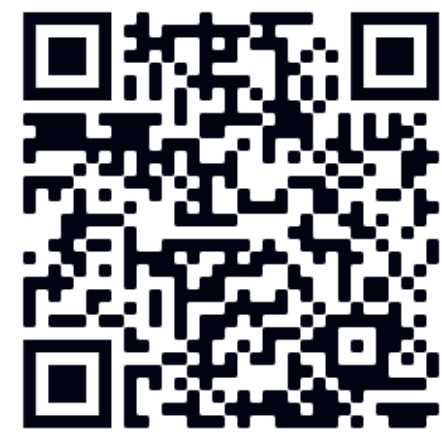

DIRECCIÓN PARA CORRESPONDENCIA:erimaryje91@ gmail.com

Fecha de recepción: 27/07/2019

Fecha de aceptación: 10/11/2019

RESUMEN

El presente proyecto, fue aplicado a los estudiantes del 9no año de Educación General Básica de la Unidad Educativa "Mariscal Sucre" del Sitio "La Codicia" del cantón Flavio Alfaro, sector rural. El objetivo principal, es que el docente pueda modificar, diseñar y realizar estrategias de enseñanza-aprendizaje con ayuda de las TICs y objetos de nuestro entorno en el área de matemáticas. Con ello, se podría lograr un ambiente armónico, que favorezca el aprendizaje significativo en los estudiantes. Por tanto, se enfatizó el tema de triángulos en el entorno y su clasificación. El enfoque principal del proyecto, es que los estudiantes tengan una ilustración de una manera más práctica y diferente, en los temas de matemática, lo cual aportaría en su aprendizaje futuro. El tema en mención, se relaciona con la vida cotidiana del estudiante, el cual permitirá que aprenda a diferenciar los tipos de triángulos que existen, según la longitud de sus lados y según la amplitud de sus ángulos, con el aporte de las TICs y con el medio que lo rodea.

PALABRAS CLAVE: ángulos; TICs; triángulos.

\section{TRIANGLES IN OUR ENVIRONMENT AND THEIR CLASSIFICATION}

\section{ABSTRACT}

The present project was applied to the students of the 9th year of General Basic Education of the Educational Unit "Mariscal Sucre" of the Site "La Codicia" of the canton Flavio Alfaro, rural sector. The main objective is that the teacher can modify, design and carry out teaching-learning strategies with the help of TICs and objects from our environment in the area of mathematics. With this, a harmonious environment could be achieved, which favors significant learning in the students. Therefore, the theme of triangles in the environment and their classification was emphasized. The main focus of the project is for students to have an illustration in a more practical and different way, in the subjects of mathematics, which would contribute to their future learning. The topic in question is related to the daily life of the student, which will allow him to learn to

\footnotetext{
${ }^{1}$ Ingeniera en Informática. Docente Unidad Educativa "Magaly Massón de Valle Carerra” Chone, Manabí y Ecuador.
} 
Erika María Muñoz Delgado,,$\ldots$

differentiate the types of triangles that exist, according to the length of their sides and the width of their angles, with the contribution of TICs and the environment that surrounds it.

KEYWORDS: angles; TICs; triangles.

\section{INTRODUCCIÓN}

Un problema radical, en la vida de todo estudiante es la complejidad en aprender sobre números, gráficos, reglas y fórmulas. Por ello, actualmente las matemáticas no son del agrado de algunos estudiantes. Es así, como el Ministerio de Educación busca mecanismos y estrategias para que el sector educativo y en especial los docentes de matemáticas, puedan impartir esta área de una manera práctica e innovadora; y con ello, poder motivar al estudiante a un mejor aprendizaje en su vida estudiantil y porque no, para la vida misma y, en su futuro próximo educativo.

Con este antecedente, algunos docentes se encuentran innovando en sus aulas de clases, para así mejorar la enseñanza-aprendizaje de hoy en día. Por ello, la autora del presente proyecto da a conocer cómo los educandos pueden aprender de una manera diferente el tema de "Triángulos en nuestro entorno y su clasificación"; el cual, ha sido una problemática para los estudiantes de 9no año de Educación General Básica de la Unidad Educativa "Mariscal Sucre", el poder diferenciar las partes de un triángulo y las clasificaciones según la longitud de sus lados y según la amplitud de sus ángulos, ha sido algo complejo, aunque el tema a simple vista, es fácil.

Por otra parte, y gracias a la existencia de las Tecnologías de Información y Comunicación (TICs), se insertan como un complemento en la vida del ser humano para el logro del aprendizaje significativo; de una u otra manera las personas se encuentran rodeadas de tecnología, al escribir un WhatsApp, al enviar un correo electrónico, navegar en la web o al instalar una aplicación en su teléfono, entre otros; es notorio que las mismas se han apoderado del ser humano y estar rodeados de ellas es inevitable.

Es sin duda alguna importante resaltar, que en el ámbito educativo son esenciales para la práctica docente porque permiten generar un sin números de ideas y recursos, para mejorar la destreza en el aula; así como la metodología de enseñanza-aprendizaje. Por ende, el presente proyecto, se basa de cómo el estudiante puede aprender con herramientas web 2.0 y recursos de su entorno durante su vida estudiantil.

\section{DESARROLLO}

La autora del presente proyecto, da a conocer que con la ayuda de las TICs y otros recursos, las matemáticas pueden ser más dinámicas y divertidas (Pabón, Nieto, \& Gómez, 2015). Por ello, el tema que se enfoca es "Triángulos en nuestro entorno y su clasificación" de una manera no tradicional, rompiendo así la brecha digital (Alva, 2015); y es el caso de algunos docentes que se rehúsan al cambio por miedo a innovar o sencillamente seguir con la modalidad de enseñanza tradicional, olvidando que todo ser humano es capaz de dar mucho más de lo que se puede imaginar, como lo indica el autor Piaget (citado por Barrón, 1993) donde manifiesta, que el ser humano es capaz de desarrollar experiencias de aprendizaje y a la vez experimentar un descubrimiento asimilativo que implica la reconstrucción de un sistema cognitivo, porque todo conocimiento emerge de una serie de conocimientos previos. 
Por lo tanto, en el reciente proyecto se incluyen a las TICs para la enseñanza y recursos del entorno, los cuales proporcionan un aprendizaje diferente con resultados acertados; más aún, en el vigente tema de "Triángulos en nuestro entorno y su clasificación". Cabe resaltar que las TICs brindan un aporte significativo en el aprendizaje, donde el estudiante asimila de una manera distinta e innovadora con ayuda del internet (Ruiz, Martínez, \& Sánchez, 2015).

En la realización del proyecto con los estudiantes del 9no año de Educación General Básica de la Unidad Educativa "Mariscal Sucre", del Sitio "La Codicia”, del cantón Flavio Alfaro, se realizaron las siguientes actividades:

\section{ACTIVIDAD 1:}

Evaluación de conocimientos previos: Esta evaluación se la realizará con la herramienta web 2.0 denominada Kahoot, la cual es una herramienta de gamificación divertida y práctica, donde se proporciona un código al estudiante para que rinda la evaluación, la cual puede ser tomada desde un computador o en su celular con cobertura de internet (Anexo 1).

Kahoot que es una herramienta gamificada de educación social; es decir, que se comporta como un juego, recompensando a quienes progresan en las respuestas con una mayor puntuación que les catapulta a lo más alto del ranking (Ramírez, 2018).

Introducción al tema: Se enfatizaron conceptos sobre el tema a tratar y se les solicito a los estudiantes que tomen referencias de problemas de la vida cotidiana, de donde encontramos los triángulos en nuestro entorno y en el mundo. Esto pudo servir para enriquecer conocimientos en los estudiantes (Anexo 2).

\section{ACTIVIDAD 2}

Aplicación de conocimientos: El estudiante reconocerá los tipos de triángulos que existen. Para ello, se llevó a efecto la elaboración de esta actividad referente al tema con la ayuda de paletas de colores, realizándolo de una manera tradicional, pero a la vez engrandeciendo sus conocimientos (Anexo 3).

Comprobación: En este apartado el estudiante realizará la comprobación de sus ejercicios (realizados con paletas) y con la ayuda de una aplicación en línea THATQUIZ con el siguiente link https://www.thatquiz.org/es-A/matematicas/triangulo/ en la cual podrá realizar las comprobaciones de los tipos de triángulos que existen y enriquecer sus conocimientos, con la ayuda de la web 2.0 (Anexo 4).

En el caso de contar con un buen laboratorio y cobertura de internet, podrán también realizar la comprobación con la ayuda de GEOGEBRA con el siguiente link https://www.geogebra.org/graphing?lang=es que es una herramienta web 2.0 utilizada para la comprobación de gráficas y en este caso de triángulos y sus clasificaciones (Anexo 4).

\section{ACTIVIDAD 3}

Se realizaron los Feedback en el tema planteado, usando estos métodos de retroalimentación.

Línea de opinión: En esta podrán acercarse a la línea +, - o +- (cinta adhesiva) de dudas y respuestas de acuerdo al tema tratado y entre los compañeros responder dudas o aportaciones de la clase.

Doble rueda: En este método se forman 2 círculos con los estudiantes y se fomentan diálogos del tema tratado de forma rotativa. 
Erika María Muñoz Delgado, , ...

Pecera: Se realizan grupos pequeños de 4 o 6 estudiantes y van participando de acuerdo a los conocimientos adquiridos en forma de debate y a la vez despejando dudas.

\section{MATERIAL Y MÉTODOS.}

Se consideró las metodologías de aprendizajes, tales como:

Metodología STEAM.- Por sus siglas en ingles Science, Technology, Engineering, Art y Mathematics, está enfocada en que los estudiantes utilicen el razonamiento a la hora de tomar decisiones; y con ello, adquieran confianza y conocimientos para que puedan desarrollar habilidades del siglo XXI. Su principal objetivo es que los estudiantes aprendan haciendo; aunque, es preciso combinar la parte práctica con la teórica utilizando varias técnicas como debates, estudiando, investigando etc. (Educación 3.0, 2018).

Aprendizaje Basado en Problemas (ABP).- Esta metodología, está basada en la resolución de problemas, donde sitúa al alumno en el centro del aprendizaje, para que sea capaz de resolver autónomamente ciertos retos o problemas (Alumnos Global, 2018). Esta metodología permite que los estudiantes identifiquen los tipos de triángulos y su clasificación dentro de la vida cotidiana.

Flipped Classroom.- Esta metodología ayuda a mejorar la enseñanza, que se genera con ayuda de las tics, creando videos, recursos multimedias, entre otros, esto es un aprendizaje significativo tanto para el estudiante como para el docente (Marqués, 2016).

\section{RESULTADOS.}

El resultado principal, es que los estudiantes de 9no año de EGB de la Unidad Educativa "Mariscal Sucre", conozcan sobre las diferentes maneras de usar la tecnología y el entorno, como un recurso educativo y formativo; y, con ello mejorar su nivel básico superior para poder enfrentarse a un bachillerato en un futuro; porque, al estar al tanto que existen herramientas y técnicas que pueden perfeccionar la enseñanza-aprendizaje de una manera divertida y poder incorporarlas en un futuro próximo, ayudará a que su nivel educativo sea superior a lo esperado; a la vez que las mismas permitirán que sus aprendizajes mejoren significativamente, sin olvidar que la ardua tarea de un docente es preparar al estudiante para la vida.

\section{DISCUSIÓN}

Es importante enfatizar que al realizar los conocimientos previos en cada clase, es de mucha ayuda para el aprendizaje del estudiante ya que se despierta la curiosidad y el entusiasmo por aprender. Una de las falencias que puede existir, es que al docente se le complique el poder observar en una cantidad de alumnos bastante extensa, problemas o vacíos en los temas impartidos; por lo que, en sectores rurales es más fácil ya que son cantidades pequeñas de estudiantes a diferencia de un sector urbano.

Existe también la ventaja de que el estudiante puede relacionar temas de matemática con el entorno y a la vez dar uso al recurso tecnológico que en la actualidad es el medio que manipulan con frecuencia, y al combinarlo con las matemáticas éste puede brindar un mejor resultado, dado que las TICs son las que aportan con un mejor aprendizaje en el estudiante.

\section{CONCLUSIONES}

La autora en el presente proyecto hace énfasis al uso de herramientas web 2.0 para mejorar la práctica en el aula con ayuda de las TICs, y como las mismas brindan el aporte necesario para su vida estudiantil y su vida cotidiana. 
- Motivar al estudiante a mejorar su enseñanza- aprendizaje dentro del aula de clases en el área de matemáticas.

- Fortalecer el conocimiento del estudiante con nuevas estrategias de aprendizaje para que sea aplicado en su diario vivir.

- Mejorar metodologías de enseñanza, aplicadas en el aula de clases para obtener mejores resultados en la práctica docente.

- Proporcionar al estudiante herramientas web 2.0 necesarias para una mejor práctica de aula y su vida cotidiana.

\section{REFERÉNCIAS BIBLIOGRÁFICAS}

Alumnos Global. (19 de 11 de 2018). APRENDIZAJE BASADO EN PROBLEMAS (ABP), METODOLOGÍA PARA EL APRENDIZAJE ACTIVO. Obtenido de https://www.alumnusglobal.com/aprendizaje-basado-enproblemas-abp-metodologia-para-el-aprendizaje-activo/

Alva, A. (2015). Los nuevos rostros de la desigualdad en el siglo xxi: la brecha digital. Revista Mexicana de Ciencias Políticas y Sociales, 265-285.

Barrón, R. (1993). Aprendizaje por descubrimiento: Principios y aplicaciones inadecuadas. Investigación y experiencias didácticas, 3-11

Educacion 3.0. (05 de 11 de 2018). 10 claves para implantar la educación en STEAM en el aula. Obtenido de https://www.educaciontrespuntocero.com/noticias/steam-en-el-aula/41064.html

Marqués, M. (2016). Qué hay detrás de la clase al revés (flipped classroom). XXII Jornadas sobre la Enseñanza Universitaria de la Informática, 77-84.

Pabón, J., Nieto, Z., \& Gómez, C. (2015). Modelación matemática y GEOGEBRA en el desarrollo de competencias en jóvenes. Revista Logos, Ciencia \& Tecnología, 65-70.

Ramírez, I. (7 de Septiembre de 2018). Kahoot!: qué es, para qué sirve y cómo funciona. Obtenido de https://www.xataka.com/basics/kahoot-que- es-para-que-sirve-y-como-funciona

Ruiz, J., Martínez, M., \& Sánchez, M. (2015). El impacto de las TICs en la calidad de la educación superior. Revista de Investigación en Ciencias Contables y Administrativas, 1(1), 1-17. 
Erika María Muñoz Delgado,,$\ldots$

32 UNESUM-Ciencias. Publicación cuatrimestral. Vol. 3, Año 2019, No. 2 (Mayo - Agosto) 